\title{
Phase matching with pulsed Bessel beams for high-order harmonic generation
}

\author{
Alessandro Averchi, ${ }_{1}^{1}$ Daniele Faccio, ${ }^{1, *}$ Ricardo Berlasso, ${ }^{1}$ Miroslav Kolesik, ${ }^{2}$ Jerome V. Moloney, ${ }^{2}$ \\ Arnaud Couairon, ${ }^{3}$ and Paolo Di Trapani ${ }^{1,4}$ \\ ${ }^{1}$ CNISM and Department of Physics and Mathematics, University of Insubria, Via Valleggio 11, 22100 Como, Italy \\ ${ }^{2}$ ACMS and Optical Sciences Center, University of Arizona, Tucson, Arizona 85721, USA \\ ${ }^{3}$ Centre de Physique Théorique, CNRS, École Polytechnique, F-91128, Palaiseau, France \\ ${ }^{4}$ Department of Quantum Electronics, Vilnius University, Sauletekio Avenue 9, bldg. 3, LT-10222, Vilnius, Lithuania
}

(Received 17 September 2007; published 20 February 2008)

\begin{abstract}
We propose a different approach to obtain phase-matched generation of high-order harmonics based on the use of pulsed Bessel beams as pump pulses. By means of the "coherence map" technique, we show that it is possible to maximize the generation of a chosen harmonic of interest by properly adjusting the phase front tilt of the pulsed Bessel beam to compensate the mismatch arising from material and plasma dispersion and atomic phase.
\end{abstract}

Since the 1990s it is well known that a crucial requirement for high-order harmonic generation (HHG) is phase matching (PM) between the pump and the harmonic field $[1,2]$. Many efforts have been made and different techniques have been proposed to overcome the mismatch factors which afflict the process and severely reduce its efficiency. The phase mismatch in HHG $\Delta k$ on axis can be expressed as $[3,4]$

$$
\begin{aligned}
\Delta k=q k_{\text {laser }}-k_{q}= & \frac{q 2 \pi(1-\eta)}{\lambda} \Delta n+P \eta N_{\text {atm }} r_{e}\left(\frac{\lambda}{q}-q \lambda\right) \\
& +(\text { geometric term })+(\text { atomic phase })
\end{aligned}
$$

where $q$ is the harmonic order, $\lambda$ is the laser wavelength, $P$, $\eta, N_{\text {atm }}, r_{e}$ are the gas pressure in atmospheres, the ionization fraction, the gas density at $1 \mathrm{~atm}$, and the classical electron radius, respectively, and $\Delta n=n_{\text {laser }}-n_{q}$ with $n_{\text {laser }}$ the refractive index for the laser and $n_{q}$ for the harmonic. The first (positive) and second (negative) terms refer to material dispersion and plasma dispersion, respectively; the geometric term has positive sign for the focused Gaussian geometry and negative in a waveguided [5] or self-guided [6] geometry, while the sign of the atomic dipole phase term (related to the quantum paths of the electrons involved in the HHG process) is in general not fixed during propagation since it is proportional to $d I / d z$ [7].

Once phase matching is established, the ultimate limit for the harmonic generation at a given laser intensity is determined by the reabsorption of the harmonic by the gas itself [8]. This condition, known as "absorption-limited HHG" is resonably fulfilled when $L_{c o h} \geqslant 10 L_{a b s}$, where $L_{c o h}$ is the coherence length, i.e., the distance over which the harmonic signal builds up coherently and $L_{a b s}$ is the absorption length in the gas. Although in literature there exist some works where this regime has been reached [9], this remains limited to very specific operating conditions, and phase-matching

\footnotetext{
*daniele.faccio@uninsubria.it
}

techniques for HHG are still an open and debated topic, as a number of recent works show (e.g., Refs. [4,10-12]).

In this Rapid Communication we address the working regime where the material dispersion term is greater than the plasma dispersion term, i.e., below the so-called "critical ionization" limit [13]. Hollow waveguides have been shown to give rise to phase matching in this regime, however their application is limited to low gas pressures and they can compensate only a relatively small phase mismatch: moreover, coupling at the input of the waveguide limits the maximum pump energies and energy loss is unavoidable. Conversely, we theoretically show how the appropriate choice of a pulsed Bessel beam (PBB) as a pump pulse permits one to achieve good phase matching for virtually any operating condition, i.e., any gas and harmonic of interest, even at high pressures (e.g., 1 atm).

Pulsed Bessel beams [14] are peculiar wave packets in which the pulse wave vectors are aligned along a conical surface such that the resulting interference pattern is characterized by a central intense peak (Fig. 1). PBBs may be efficently generated by sending a laser pulse through an axicon or a suitable hologram. Two properties of PBBs make them promising for HHG: first, compared to the Rayleigh range of a Gaussian pulse having the same width, the PBB is nondiffractive over a much longer distance (known as "Bessel zone"). This in turn implies a propagation with a smoother intensity and phase evolution [15] and a great stabilization of

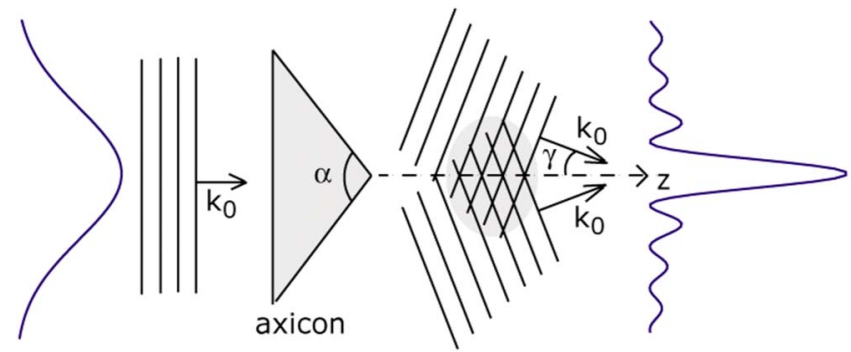

FIG. 1. (Color online) Bidimensional sketch of the generation of a PBB by sending a Gaussian pulse into an axicon. The phase fronts acquire a tilt $\gamma$. The interference between the off-axis propagating fronts creates the peculiar radial intensity distribution. 
the fluctuations in the atomic phase term in Eq. (1) which may be caused by oscillations in the pump pulse intensity and have a detrimental role for phase matching. The second crucial property of $\mathrm{PBB}$ is the possibility to control the phase velocity by simply changing the angle of the Bessel cone. In particular, the axial wavevector $k_{z}$ will always be shortened with respect to the plane wave $k_{0}$ by an amount $\beta$ given by $\beta=k_{0}(1-\cos \gamma)$, where $\gamma$ is the angle of the Bessel cone. Substituting $k_{z}=k_{0}-\beta$ into Eq. (1), we obtain for the PBB

$$
\begin{aligned}
\Delta k=q k_{\text {laser }}-k_{q}= & q\left(k_{0}-\beta\right)-k_{q} \\
= & \frac{q 2 \pi(1-\eta)}{\lambda} \Delta n-P \eta N_{a t m} r_{e} q \lambda \\
& +(\text { atomic phase })-q \beta,
\end{aligned}
$$

where we have neglected the plasma dispersion relative to the harmonic signal (e.g., Ref. [5]). The peculiar contribution given by $-q \beta$ has the same sign as the plasma dispersion term. Therefore, for pump intensities below the critical ionization threshold one can think to choose a suitable $\beta$ (i.e., a suitable axicon or a hologram pattern) to compensate the phase mismatch between pump and signal. We note that the contribution to phase matching given by $-q \beta$ plays the same role as the waveguide geometric term, but in a much wider range: in waveguides the contribution $\Delta k$ scales as $1 / a^{2}$, and with a diameter $a=100 \mu \mathrm{m}, \Delta k \approx 36 \mathrm{~m}^{-1}$. In contrast, with a simple axicon having, for example, $\beta=2.8^{\circ}$ one obtains $\Delta k$ $\approx 2337 \mathrm{~m}^{-1}$. A similar $\Delta k$ in the hollow waveguide configuration would require use of a very narrow waveguide with $a=12 \mu \mathrm{m}$ with extremely difficult alignment and energy coupling.

To investigate phase matching with $\mathrm{PBB}$, we adopted the well-established technique of the "coherence map," introducted by Balcou et al. in [7]. Following the same approach as Tosa et al. in Ref. [6] we applied this technique to the calculated solution of the pump propagation equation, which was obtained by numerically solving the nonlinear Schrödinger equation (NLSE, for a detailed description of the code see, e.g., Ref. [16]) taking into account all the effects-such as plasma generation and material dispersion-which play a role in the phase matching. Ammosov-Delone-Krainov (ADK) rates [17] were used to estimate the tunneling ionization. With this procedure all the effects that have a role in the phase matching are included in the calculation and there is no need to consider them separately.

We recall very briefly to the reader that the coherence map gives the coherence length $L_{c o h}$ for the generation process of a given harmonic order $q$, along the radial profile of the pump pulse at its intensity peak. The coherence length describes the propagation length over which the signal adds up constructively and is defined as $L_{c o h}=|2 \pi / d k|$, where $d k$ $=2 \pi q n_{q} / \lambda-\left|\mathbf{k}_{\text {pol }}\right| ; n_{q}$ is the refractive index for the harmonic signal (taken from Ref. [19]), which allows us to take into account in our calculation the material dispersion at the harmonic wavelength, and $\mathbf{k}_{\text {pol }}$ is the polarization wavevector given by $\mathbf{k}_{\mathrm{pol}}=q \boldsymbol{\nabla}[\phi(r, z)]+\boldsymbol{\nabla}[\alpha I(r, z)]$. Here $\phi(r, z)$ and $I(r, z)$ are the fundamental field phase and intensity profile

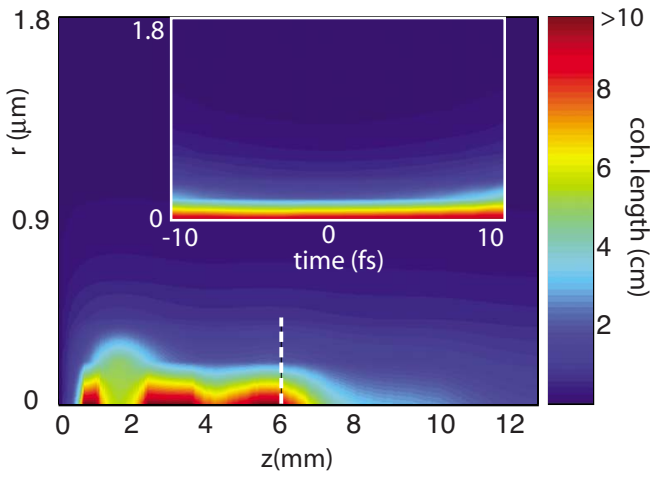

FIG. 2. (Color online) Coherence length (in $\mathrm{cm}$ ) for a Bessel pump beam in helium phase matched with the 61st harmonic over $1 \mathrm{~cm}$ of propagation from the beginning of the gas cell. The inset shows the radial vs local time coherence map along the PBB profile at $z=6 \mathrm{~mm}$ (dashed line).

retrieved by the simulation and $\alpha$ is the phase coefficient for the harmonic field [18].

In order to illustrate the impact of the phase-matching capability of PBBs we studied the generation of harmonic 61 st in helium at relatively low pump intensities. This regime allows us to clearly illustrate the role of PBB-induced phase matching, as the contribution due to plasma dispersion is relatively small. To compare the harmonic yield with that of a conventional Gaussian pulse we proceeded in the following way: for both pulses we fixed the same duration, energy, and peak intensity, taking typical experimental conditions of, respectively, 35 fs full width at half maximum (FWHM), $5 \mathrm{~mJ}$, and $4 \times 10^{14} \mathrm{~W} / \mathrm{cm}^{2}$, which is an intensity slightly above the cutoff value for the 61st harmonic. Keeping these parameters always fixed, we optimized our beam with an iterative procedure by varying the PBB angle and studying the corresponding coherence maps for the 61st harmonic. We obtained the best phase matching conditions for the PBB with a cone angle of $2.8^{\circ}$, which in turn determined also the diameter of the central peak as $31 \mu \mathrm{m}$ between the first zeros and the $1 / e^{2}$ radius of the Gaussian apodization of the beam, $86 \mu \mathrm{m}$. The Gaussian beam we used for comparision had a $1 / e^{2}$ radius $w_{0}=113 \mu \mathrm{m}$, uniquely determined by energy, duration, and peak intensity. We chose to work at a pressure $P=1 \mathrm{~atm}$, with an absorption length for the 61st harmonic in helium $L_{\alpha} \sim 1.46 \mathrm{~mm}$ [19]. Therefore to reach the absorption limit it is sufficient to optimize the PBB $\beta$ so as to obtain a coherence length of a few centimeters.

Figure 2 shows the calculated coherence length map for the given parameters which correspond to the result of the optimization process: Since the harmonic 61st is in the cutoff region for our chosen intensity, the phase coefficient for the atomic potential is $\alpha=-13.7 \times 10^{-14} \mathrm{~cm}^{2} / \mathrm{W}$ [18]. As it may be seen, the coherence map exhibits a sharp peak only $\sim 0.5 \mu \mathrm{m}$ wide and distributed along the propagation direction $z$ in which the coherence length $L_{c o h} \geqslant 3 \mathrm{~cm}$. As a result of the nondiffractive nature and nearly stationarylike propagation of the $\mathrm{PBB}$, this value is maintained over a large propagation distance.

In the inset of the same figure we also show the coherence length along the spatiotemporal $(r, t)$ pulse profile, for a fixed 


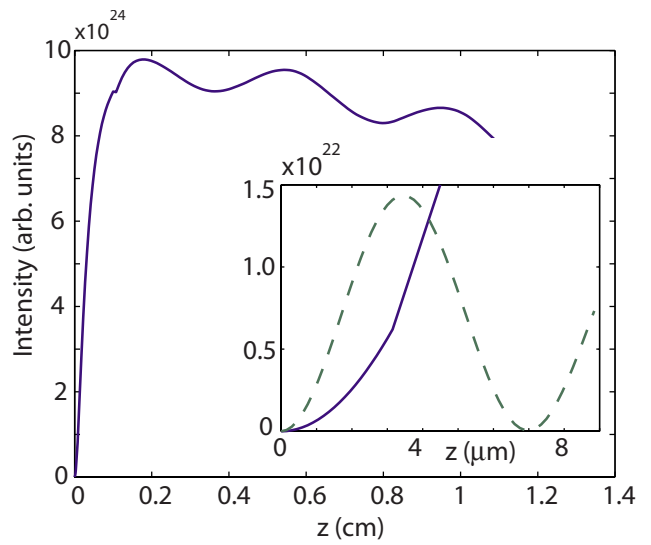

FIG. 3. (Color online) Calculated power in the 61st harmonic generated in helium using a phase matched PBB pump. The inset shows in detail the power growth in the first few microns compared to that obtained with the Gaussian pump pulse with the same input energy and peak intensity.

position on the propagation axis $z=0.6 \mathrm{~cm}$ (dashed line in Fig. 2). The values of $L_{c o h}$ remain almost constant along the pulse temporal axis, so that the harmonic field builds up coherently over the pump pulse as long as the intensity is high enough.

To study the harmonic power yield, we made an analytical estimation based on Eq. (1) in Ref. [8] by assuming as in Refs. [20,21] the atomic response at the harmonic frequency $A_{q}$ to be proportional to the pump intensity $I_{l}$ as $A_{q} \propto I_{l}^{p}$, where $p=5$ is a constant value. We note that this method gives a useful indication of the relative strengths of the harmonic signals generated by the PBB and the Gaussian pulse, but it is unable to predict the effective efficiency conversion, which would require a full simulation of the HHG process.

The result can be seen in Fig. 3, which shows the evolution of the harmonic power (intensity integrated over the effective area in which the harmonic is generated). The harmonic power steadily increases until the absorption limit is reached around $z=2 \mathrm{~mm}$. At this point the harmonic yield saturates and, following the behavior of the PBB, undergoes some oscillations around the maximum value and then starts slowly to decrease when the PBB approaches the end of the Bessel zone and starts diffracting. The inset to Fig. 3 shows a detailed comparison of the harmonic power obtained by the $\mathrm{PBB}$ and Gaussian pulse with the same input duration, energy, and peak intensity of the PBB. The Gaussian pulse evolution was estimated using the same NLSE model as for the PBB, so that all nonlinear effects are accounted for. Due to the larger width of the equivalent Gaussian pulse within which the conditions for generation of the 61st harmonic are satisfied (the useful diameter for HHG is $76 \mu \mathrm{m}$ ), the initial yield of the Gaussian pulse is higher than that of the PBB. However the coherence length for a nonphase matched process in this regime is only $\sim 3 \mu \mathrm{m}$, so that the conversion process quickly reaches a maximum value followed by quick oscillations (due to the phase mismatch) and relaxes on a lower value, about one third of the maximum value in the inset, determined by the reabsorption process [8]. So, even comparing the $\mathrm{PBB}$ conversion efficiency with the maximum

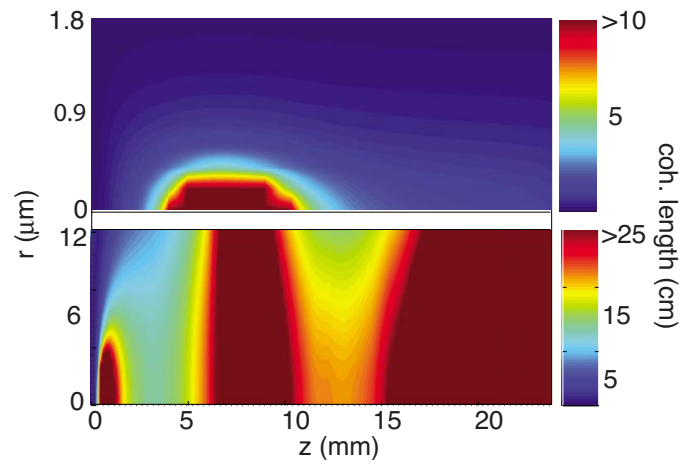

FIG. 4. (Color online) Coherence length (in $\mathrm{cm}$ ) for the 47th harmonic. The long path (top) is phase matched only in a small region on axis, while the short path (bottom) coherence length is always over $10 \mathrm{~cm}$. Note the different radial scales.

value obtained by the Gaussian pulse (at $z=5 \mu \mathrm{m}$ ), and even if the spot size over which the harmonic is generated is only around $10^{-5}$ of the Gaussian case, we still have a factor greater than 700 improvement thanks to optimal phase matching and to the fast nonlinear growth of the HHG signal versus $z$.

We verified also the effects of PBB-induced phase matching well within the harmonic plateau region. In Fig. 4 we show the coherence length maps obtained for the 47th harmonic with the same pump pulse after a reoptimization of the cone angle, which gave a slightly different value of $2.84^{\circ}$. Since we are now in the plateau region, we have two different coherence maps corresponding to the short $(\alpha=0)$ and long $\left(\alpha=-24.8 \times 10^{-14} \mathrm{~cm}^{2} / \mathrm{W}\right)$ [7] paths. The coherence map of the short path exhibits very high values of the coherence length (15 cm and more) wherever the intensity is high enough for the generation process. The long path is instead well phase matched only in a narrow region on axis. This shows that in our phase-matching scheme the atomic phase plays a critical role in limiting the spatial width of the harmonic beam so that in the cutoff region or for long path contributions in the plateau we expect to always obtain in the near field a narrow harmonic beam. By repeating for the 47 th harmonic the comparison with the Gaussian pulse, we found for the long path an improvement of a factor 100 and for the short path a much higher factor 25000 thanks to the wider radius of the phase-matched region. Before concluding, we should note that this technique has analogies with a proposed method where the interference between two beam crossing at an angle serves as a pump pulse [22]; however, beside the obvious advantages of a single beam setup, the use of PBBs avoids the poor spatial quality of the harmonic field that is almost unavoidable in the two-beam arrangement, where the interference pattern would be transferred to the harmonic unless the two beams are as narrow as a few tens of microns (so as to obtain a single interference peak), which in turn would imply a very short overlap and copropagation. We may compare this situation with PBB-induced phase matching in conditions similar to those adopted in Ref. [22], i.e., lower-order harmonic generation in argon gas at $P=0.2$ atm with a pump energy of $2.5 \mathrm{~mJ}$ and a peak intensity $I=1.6 \times 10^{14} \mathrm{~W} / \mathrm{cm}^{2}$. Figure 5 shows the result for the 27th and 29th harmonics, 


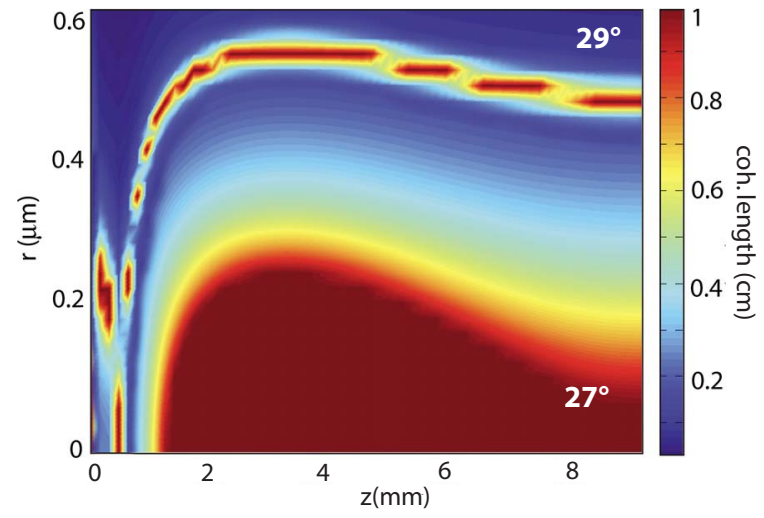

FIG. 5. (Color online) Coherence lenghts for the 27th and 29th harmonics in argon simultaneously phase matched, respectively, on axis and off axis. The coherence length scale for the 29th harmonic should be multiplied by 10 .

which are simultaneously phase matched over very long propagation distances. We underline the very different spatial distribution of the two harmonics, which would allow one to select one or the other with a spatial filter.

In conclusion, we have shown that by appropriate choice of the PBB parameters it is possible to effectively phase match the HHG and reach the absorption limit for any har- monic of interest (as long as the pump pulse remains below the critical ionization threshold), a result that can be reached only in very specific operating conditions using Gaussian pulses, and without the limitations of a hollow waveguide. Moreover, since the harmonic signal remains high over a reasonable distance of several millimeters once it reaches the absorption limit, the experimental realization with a gas cell should easily be feasible. This technique is studied in specific cases in $\mathrm{He}$ and Ar gases but may be readily applied in other operating conditions, in particular to higher pump intensites (while remaining below the critical ionization limit).

Our results within the approximation of the coherence map technique give a strong indication of a new possible approach for phase matching that we believe deserves further attention.

The authors acknowledge support from the Consorzio Nazionale Inter-universitario per le Scienze della Materia (CNISM), progetto INNESCO. P.D.T. acknowledges support from Marie Curie Chair project STELLA, Contract No. MEXC-CT-2005-025710. M.K. and J.V.M. acknowledge support from the U.S. Air Force Office for Scientific Research under Grant No. FA9550-07-1-0010. J.V.M. acknowledges support from the Alexander von Humboldt Foundation.
[1] A. L'Huillier, K. J. Schafer, and K. C. Kulander, Phys. Rev. Lett. 66, 2200 (1991).

[2] P. Balcou and A. L'Huillier, Phys. Rev. A 47, 1447 (1993).

[3] T. Pfeifer, C. Spileman, and G. Gerber, Rep. Prog. Phys. 69, 443 (2006).

[4] A. Paul, E. Gibson, X. Zhang, A. Lytle, T. Pomintchev, X. Zhou, M. Murnane, I. Christov, and H. Kapteyn, IEEE J. Quantum Electron. 42, 14 (2006).

[5] C. G. Durfee, A. R. Rundquist, S. Backus, C. Herne, M. M. Murnane, and H. C. Kapteyn, Phys. Rev. Lett. 83, 2187 (1999).

[6] V. Tosa, E. Takahashi, Y. Nabekawa, and K. Midorikawa, Phys. Rev. A 67, 063817 (2003).

[7] P. Balcou, P. Salieres, A. L'Huillier, and M. Lewenstein, Phys. Rev. A 55, 3204 (1997).

[8] E. Constant, D. Garzella, P. Breger, E. Mevel, Ch. Dorrer, C. Le Blanc, F. Salin, and P. Agostini, Phys. Rev. Lett. 82, 1668 (1999).

[9] M. Schnürer, Z. Cheng, M. Hentschel, G. Tempea, P. Kalman, T. Brabec, and F. Krausz, Phys. Rev. Lett. 83, 722 (1999).

[10] H. Kapteyn, O. Cohen, I. Christov, and M. Murnane, Science 317, 775 (2007).

[11] J. Painter, M. Adams, N. Brimhall, E. Christensen, G. Giraud,
N. Powers, M. Turner, M. Ware, and J. Peatross, Opt. Lett. 31, 3471 (2006).

[12] H. S. Chakraborty, M. B. Gaarde, and A. Couairon, Opt. Lett. 31, 3662 (2006).

[13] A. Rundquist, C. G. Durfee, S. Backus, C. Heme, Z. Chang, M. M. Murnane, and H. C. Kapteyn, Science 280, 1412 (1998).

[14] Z. Liu and D. Fan, J. Mod. Opt. 45, 17 (1998).

[15] C. Altucci, R. Bruzzese, D. D'Antuoni, C. de Lisio, and S. Solimeno, J. Opt. Soc. Am. B 17, 34 (2000).

[16] A. Couairon and A. Mysyrowicz, Phys. Rep. 441, 47 (2007).

[17] M. V. Ammosov, N. B. Delone, and V. P. Krainov, Sov. Phys. JETP 64, 1191 (1986).

[18] M. Lewenstein, P. Salières, and Anne L'Huillier, Phys. Rev. A 52, 4747 (1995).

[19] www-cxro. lbl. gov

[20] Y. Tamaki, J. Itatani, M. Obara, and K. Midorikawa, Phys. Rev. A 62, 063802 (2000).

[21] J. L. Krause, K. J. Schafer, and K. C. Kulander, Phys. Rev. A 45, 4998 (1992).

[22] S. V. Fomichev, P. Breger, B. Carre, P. Agostini, and D. F. Zaretsky, Laser Phys. 12, 303 (2002). 